\title{
A tabular source approach to modelling and simulating device and circuit noise in the time domain
}

\author{
M. E. Brinson ${ }^{1 *}$, S. Jahn ${ }^{2}$ and H. Nabijou ${ }^{3}$ \\ ${ }^{1}$ Centre for Communications Technology, London Metropolitan University, London N7 8DB, U.K. \\ 2 Qucs project manager, Munich, Germany. \\ ${ }^{3}$ Centre for Communications Technology, London Metropolitan University, London N7 8DB, U.K.
}

\begin{abstract}
SUMMARY
Simulation of device and circuit noise at low frequencies is often done as part of small signal ac analysis. Moreover, circuit simulators with rf analysis capabilities usually specify circuit performance in terms of $\mathrm{S}$ parameters and model high frequency noise in terms of noise waves and correlation matrices. It is also unusual to find circuit simulators that extend noise simulation to the time domain. This is particularly true for software packages developed from SPICE $2 \mathrm{~g} 6$ or 3f5. This paper introduces a simple tabular noise source technique which adds time domain noise to semiconductor device models and integrated circuit macromodels. The proposed technique is suitable for use with any general purpose circuit simulator. To demonstrate the power of the suggested approach the text describes time domain noise extensions to the SPICE diode, BJT, JFET, MOSFET and MESFET models. These noise extensions have been implemented and tested with the "Quite universal circuit simulator" (Qucs). Copyright (c) 2010 John Wiley \& Sons, Ltd.
\end{abstract}

KEY WORDS: Tabular noise sources; thermal, shot and $1 / f$ noise, mixed-mode equation-defined noise macromodels; Qucs: circuit simulation; Verilog-A

\section{INTRODUCTION}

Computer aided noise analysis is important in the design of analogue and microwave integrated circuits. The popular SPICE $2 \mathrm{~g} 6$ and $3 \mathrm{f} 5$ circuit simulators [1], [2] use dc non-linear component linearisation plus small signal ac analysis to determine noise. In this technique individual noise contributions are modelled by small signal ac current sources connected at a point in a circuit where the noise originates. The resulting signals propagate through a linearised circuit, appearing at an output as a signal equivalent to noise with modified spectral density. Due to the fact that individual noise contributions are uncorrelated, the total output power spectral density becomes the sum of the power spectrum densities resulting from each noise component. In microwave integrated circuit design it has become common practice to specify the circuit

${ }^{*}$ Correspondence to: M. E. Brinson, Centre for Communications Technology, London Metropolitan University, London N7 8DB. E-mail: mbrin72043@yahoo.co.uk.

Copyright (C) 2010 John Wiley \& Sons, Ltd. 
properties of an $\mathrm{n}$ port network in terms of $\mathrm{S}$ parameters and to express circuit noise as noise waves, where the noise characteristics are defined by an outgoing noise wave from each port and a matrix representing the correlation between noise sources [3]. Unfortunately, both small signal ac and S parameter noise analysis techniques are applicable only to the simulation of circuits in the frequency domain, implying that alternative analysis methods are required for the simulation of analogue and mixed-mode circuit noise in the time domain. A number of such techniques have been reported in the published literature. These are often based on the solution of stochastic differential algebraic equations [4],[5]. In general however, there would appear to be no universally established practice for modelling time domain noise among the open source General Public License (GPL) and commercial circuit simulators. Moreover, a high percentage of circuit simulators developed from SPICE $2 \mathrm{~g} 6$ or $3 \mathrm{f} 5$ make no attempt to simulate circuit noise in the time domain. This paper introduces a simple tabular noise source technique which allows time domain noise properties to be added to existing and new device models and circuit macromodels. The proposed technique is suitable for use with any general purpose circuit simulator which allows subcircuits, equation-defined components (or Verilog-A code models) and a text file (or a net-list statement) for listing tabulated voltage and current source noise data. To demonstrate the power of the proposed approach the paper describes extensions to the SPICE diode, BJT, JFET, MOSFET [6] and MESFET [7] models which add time domain noise properties to the published SPICE models. These models have been implemented and tested with the GPL "Quite universal circuit simulator" (Qucs) [8].

\section{TABULAR TEXT FILES AS VOLTAGE AND CURRENT NOISE SOURCES}

The analysis of noise in the time domain can be undertaken with transient simulation provided component or circuit noise is modelled by time dependent voltage or current sources which generate random signals with the correct magnitudes and spectral distributions. During transient simulation, at each time step, injected noise perturbs circuit voltage and current signals, leading to output waveforms which have noise components superimposed on the signals being processed. Circuit simulators that do not incorporate time dependent noise operate essentially in a noise free environment which leads to output waveforms that exhibit ideal signal characteristics rather than those observed with measured signals. Time domain noise simulation is not intended to replace small signal ac or $\mathrm{S}$ parameter noise analysis but is introduced as an additional CAD technique which allows the effects of noise to be observed on ac time domain and mixed-mode signal waveforms, particularly at small signal levels commonly found in low noise circuits. The Qucs circuit simulator is one of a number of General Public License (GPL) software packages which have become very popular amongst the science and engineering communities for rf and non-linear transient domain simulation. A second highly respected GPL program is the numerical mathematics package Octave [9]. By linking Qucs and Octave it becomes possible to use Octave programming features and numerical data processing facilities to firstly generate Qucs time dependent tabular voltage and current source data files or secondly to post-process Qucs simulation output data. Shown in Figure 1 are the schematics for Qucs file type voltage and current generators [10], where generator output voltage $\bar{v}^{2}(t)$ and current $i^{2}(t)$ are normalised functions of white noise pseudo random distribution $\xi_{n}(t)$ and 1 /f noise pseudo random distribution $\xi_{f}(t)$. The equations listed in Table I indicate how 
(a)

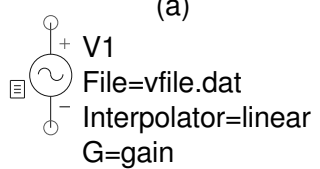

(b) 11

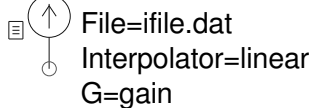

Figure 1. Qucs text file based signal generator schematics: (a) voltage; (b) current.

Table I. Thermal, shot and $1 /$ f noise source equations

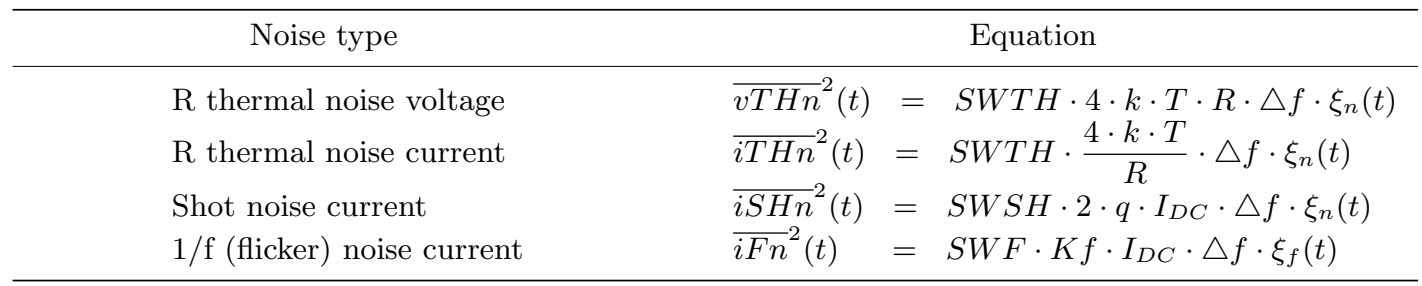

Where $\triangle f$ is the noise frequency bandwidth in $\mathrm{Hz}, \mathrm{R}$ is a resistance value in $\Omega, \mathrm{k}$ is Boltzmann's constant, $\mathrm{T}$ is temperature in Kelvin, $\mathrm{q}$ is the electron charge, $I_{D C}$ is dc current in $\mathrm{A}$, and $\mathrm{Kf}$ is a $1 / \mathrm{f}$ noise coefficient. $\xi_{n}(t)$ is a white noise sequence (values against time) with a pseudo random distribution having zero average value and a variance of one. $\xi_{f}(t)$ is a normalised noise sequence with a $1 / f^{A f}$ pseudo random distribution with Af a 1/f noise exponent. Parameters SWTH, SWSH and SWF act as on-off switches; when 1 individual noise contributions are turned on, when 0 contributions are off.

time dependent thermal, shot and 1/f (flicker) noise sources can be formed from $\xi_{n}(t)$ and $\xi_{f}(t)$. Qucs allows linear, hold or spline interpolation of tabular data with the data sequence either repeating, when its end is reached, or held at its final value for all future values of time. Qucs files "vfile.dat" and "ifile.dat" list the tabulated data and its associated time values in seconds. A basic Octave $m$ script is given in Table II which is suitable for generating Qucs "xx.dat" text files [11].

\section{MODELLING THERMAL NOISE IN THE TIME DOMAIN}

Figure 2 shows a simple time domain test circuit for recording the thermal noise [12] generated by a number of different resistors. The resistor noise model consists of a tabular voltage generator, formed from one million pseudo random white noise voltage samples taken over a one second period, in series with a noise free resistor. Qucs equation block Eqn5 is used to calculate the voltage source parameters prior to the start of a transient simulation. In this context variable Scale_factor defines a scaling constant which is a function of the noise rms to peak conversion crest factor ( 1 to approximately 3.2 depending on the type of noise [13]). Scale_factor supports scaling of the Qucs generated noise values to fit theoretical or measured noise data. Similarly, equation script Eqn1 forms part of the transient simulation control instructions. This script is also actioned before the start of transient simulation. Post transient simulation data analysis is undertaken by the code listed in Qucs equation block Eqn6. Eqn6 calculates the rms values of the resistor noise voltages from the Qucs time domain 
Table II. Octave m script for generating Qucs "xxx.dat" noise data files.

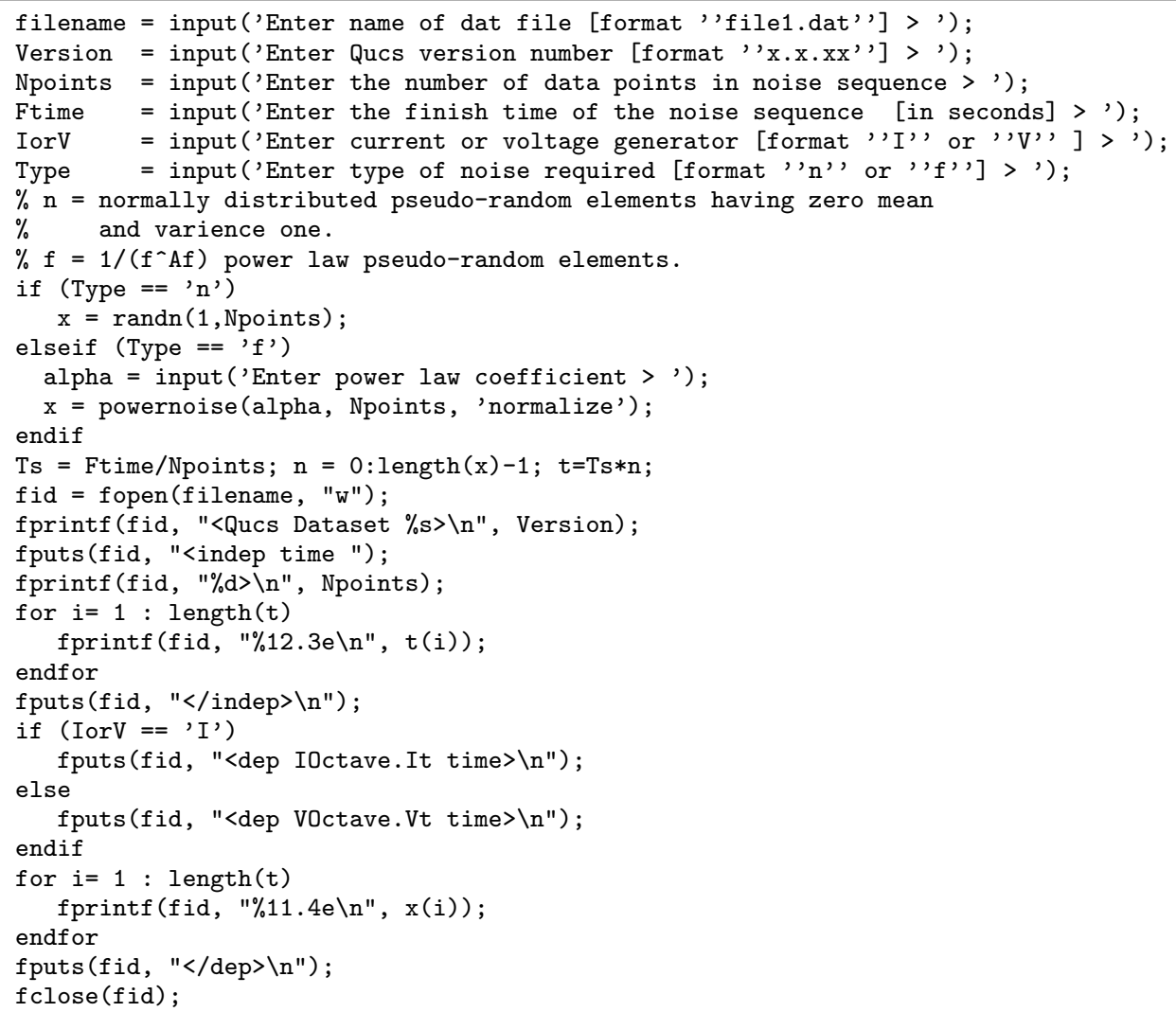

Octave function "powernoise" generates $1 / f^{A f}$ pseudo random sequences, where Af $\approx 1[16]$.

simulation output data. The waveforms plotted in Figure 2 show the time domain noise data generated by Qucs. Waveform inserts give values for both the Qucs time domain and ac small signal voltage rms values obtained from simulation. Clearly this data confirms agreement between the time and ac domain noise models. Current generator thermal noise models show similar behaviour. Careful inspection of the waveforms in Figure 2 also confirm that the four noise voltage generator signals are uncorrelated.

\section{MODELLING SHOT NOISE IN THE TIME DOMAIN}

Figure 3 introduces a tabular time domain model for transient simulation of current shot noise [12]. In Figure 3(a) a schematic symbol for the shot noise subcircuit is drawn where the lefthand side of the circuit is used to sense the dc current generating the shot noise, and $\xi_{n}(t)$ and $i S H n$ are as defined in Table I. The body of the time domain shot noise model is given in Figure 3(b). Resistor $R x$ and capacitor $C x$ act as a low pass filter that ensures the value 


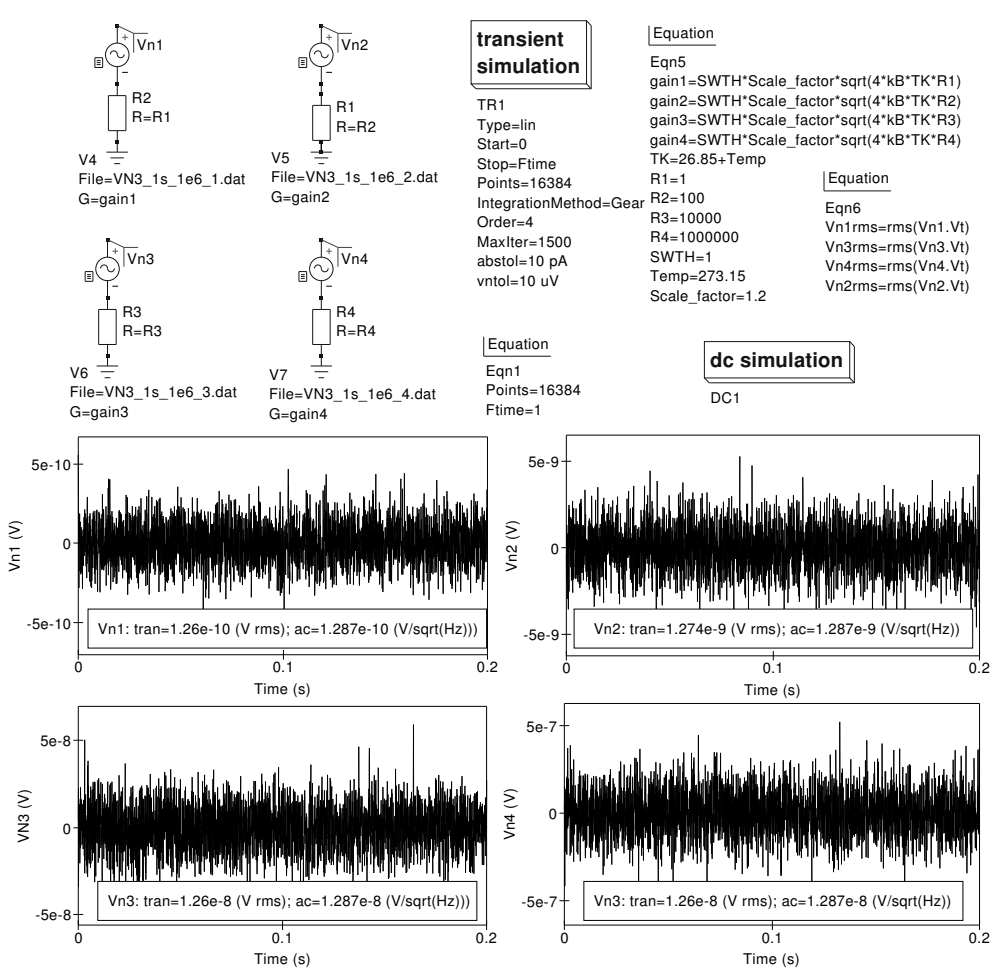

Figure 2. Time domain resistor noise test circuit, Qucs equation scripts for transient domain simulation and post processing noise waveforms: simulation period 1 second, and linear interpolation with Gaussian voltage noise variance; file VN3_1s_1e6_1.dat $=0.942 V^{2}$, file VN3_1 $\mathrm{s}_{-} 1 \mathrm{e} 6{ }_{-} 2$.dat $=0.950 \mathrm{~V}^{2}$, file VN3_1s_1e6_3.dat $=0.952 V^{2}$, file VN3_1s_1e6_4.dat $=0.958 V^{2}$, and average values $(\mathrm{dc})$; file VN3_1s_1e6_1.dat $=$ $0.0124 V$, file VN3-1 $\mathrm{s}_{-} 1 \mathrm{e} 66_{-}$.dat $=-0.00789 V$, file $\mathrm{VN}{ }_{-} \mathrm{s}_{-} 1 \mathrm{e} 6-3$. dat $=0.0111 V$, file VN3_1s_1e6_4.dat $=0.0047 \mathrm{~V}$.

of the current used to calculate shot noise is purely dc and does not contain any time varying components. Shot noise is computed during simulation by Qucs non-linear equation-defined device EDD D1 [14]. The graph plotted in Figure 3(c) presents a set of shot noise results obtained from simulation of the shot noise subcircuit model over the dc current range $1 \mu \mathrm{A}$ to 1A. Table III lists a Verilog-A [15] analogue module for the current shot noise model. The structure of the model and the basis for the current contribution statements can be identified by comparing the Verilog-A code with the Qucs schematic drawn in Figure 3(b).

\section{MODELLING $1 /$ f NOISE IN THE TIME DOMAIN}

The tabular time domain model for transient simulation of $1 / \mathrm{f}$ noise [12] is similar to the shot noise model illustrated in Figure 3. The major change required is to replace EDD D1 equation 


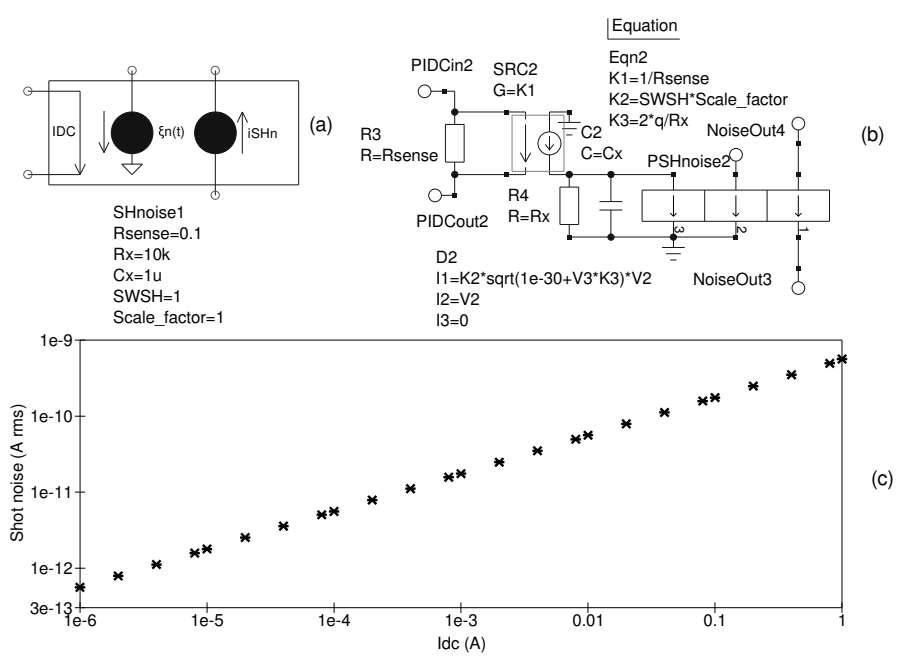

Figure 3. Time domain shot noise subcircuit model: (a) Qucs schematic symbol; (b) model body; (c) shot noise plotted against dc current.

Table III. Verilog-A code for a current shot noise time domain model.

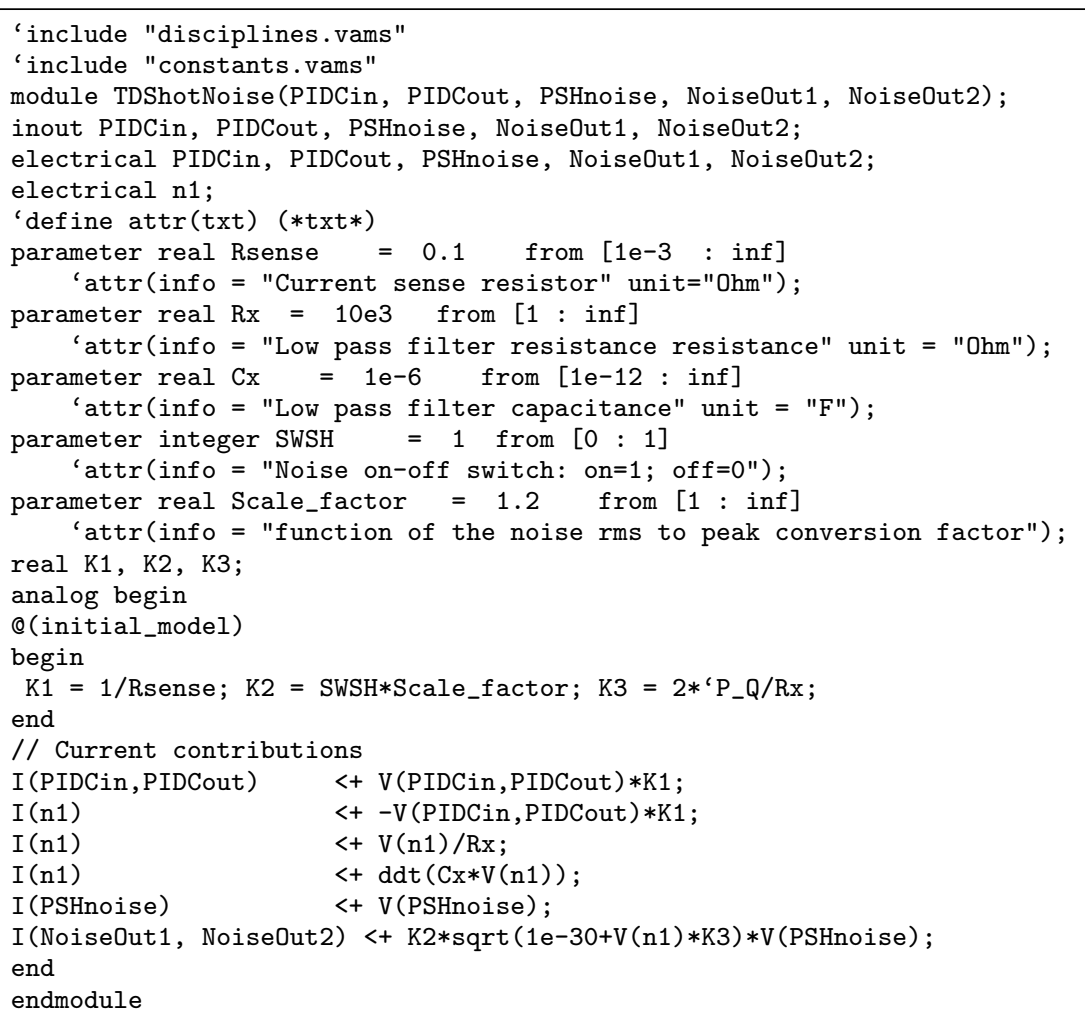




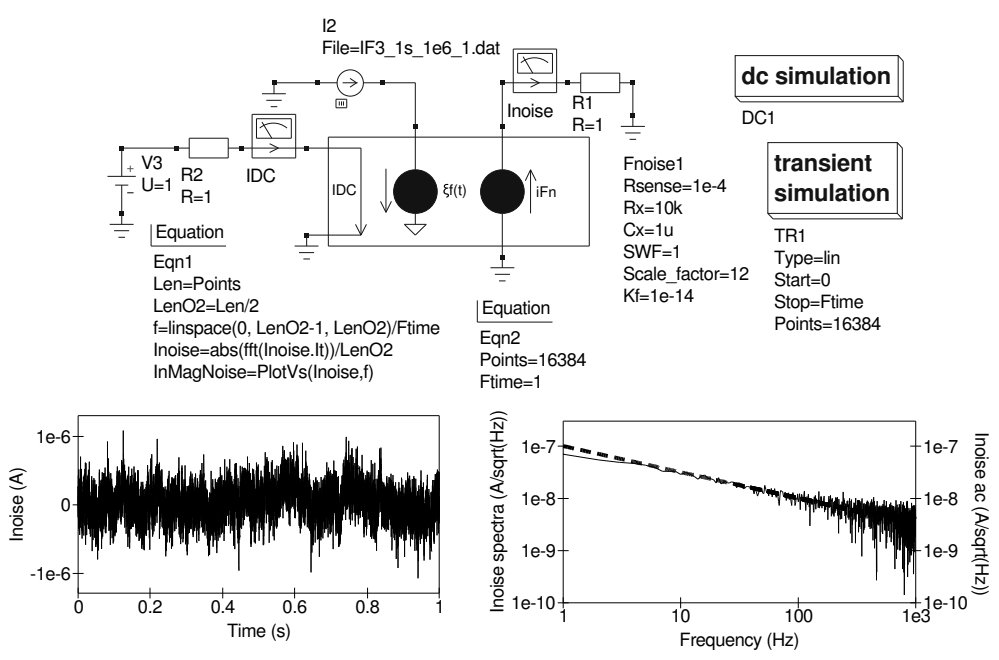

Figure 4. Time domain 1/f noise test circuit and simulation waveforms: frequency domain plot; solid line left hand scale, dashed line right hand scale.

I1 by equation 1 .

$$
I 1=K 2 * \operatorname{sqrt}(1 e-30+V 3 * K f) * V 2
$$

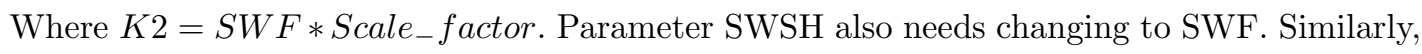
note variable $\mathrm{K} 3$ is no longer required. Corresponding changes are also needed to adapt the Verilog-A shot noise module code, including changing the Verilog-A module name to say TDFlickerNoise. Figure 4 gives a basic simulation test circuit which allows the $1 / \mathrm{f}$ noise to be recorded as a function of time. Central to the time domain $1 / \mathrm{f}$ model is a tabular current generator, I2 in Figure 4, formed from one million noise samples with normalised $1 / \mathrm{f}$ pseudo random distribution. The waveforms in Figure 4 show the generated $1 / \mathrm{f}$ noise for a period of one second. Qucs equation block Eqn1 lists a post simulation script that extracts the noise spectra of the $1 / \mathrm{f}$ time domain data. FFT techniques were used to assemble the frequency domain plot. Comparison between the time domain and the small signal ac noise data indicates good agreement. However, one feature of the shot and $1 / \mathrm{f}$ noise data is worth commenting on, namely

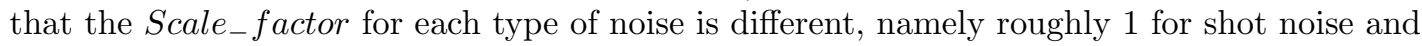
12 for $1 / \mathrm{f}$ noise. One possible explanation for this is crest factors for the two different noise sequences; with shot noise a crest factor of around 1 (Scale_factor $\approx 1.2$ ) seems correct, while for $1 / \mathrm{f}$ noise a crest factor of around $3.2\left(\right.$ Scale factor $\left.\approx 3.2^{2}\right)$ appears to be more appropriate [17].

\section{MODELLING DIODE NOISE IN THE TIME DOMAIN}

The SPICE semiconductor diode model includes small signal ac series resistive thermal noise, shunt shot noise and shunt $1 / \mathrm{f}$ noise. However, standard SPICE does not model burst (popcorn) [6] noise. The thermal noise, shot noise and $1 / \mathrm{f}$ noise contributions are given by equations 2 


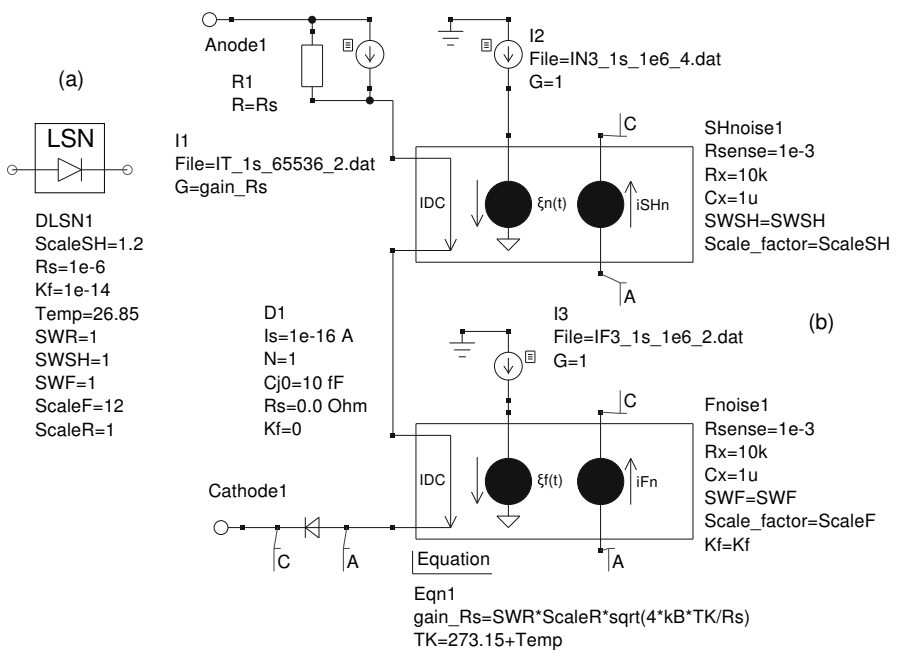

Figure 5. A semiconductor diode model with time domain noise additions: (a) Qucs schematic symbol; (b) model body.

and 3 respectively.

$$
\begin{gathered}
\bar{i}_{R S}^{2}(t)=\frac{4 \cdot k \cdot T}{R S} \cdot \Delta f \cdot \xi_{n}(t) \\
\bar{i}_{D}^{2}(t)=2 \cdot q \cdot I_{D} \cdot \Delta f \cdot \xi_{n}(t)+K f \cdot \frac{I_{D}}{f^{A f}} \cdot \Delta f \cdot \xi_{f}(t)
\end{gathered}
$$

Where $I_{D}$ is the diode dc current in $\mathrm{A}$ and $R S$ is the diode series resistance in $\Omega$. The other symbols have the same meaning as previously defined. Time domain equivalent noise components can be added to the SPICE diode model by combining the published diode model with the tabular noise models introduced in previous sections. Figure 5 gives such a model. In Figure 5 a resistance $R S$ is connected in series with the shot and $1 / \mathrm{f}$ noise generation components and a standard SPICE diode (D1), ensuring that the dc current flows between the Qucs diode subcircuit anode and cathode terminals. The diode internal value of RS is set to zero to ensure that only one copy of $R S$ is included in the large signal diode noise model. The simulation test results illustrated in Figure 6 confirm good agreement between the small signal ac and time domain noise models. Again a best fit value for the $1 / \mathrm{f}$ Scale_factor was found to be approximately $3.2^{2}$, suggesting that the $\mathrm{rms}$ to peak crest factor for $1 / \mathrm{f}$ noise signals is roughly 3.2. With SWF set to 0 the rms value of the diode shot noise can be extracted from the time domain diode noise waveform, yielding a figure of 5.592e-10 A rms which is very close to the theoretical value of $5.762 \mathrm{e}-10 \mathrm{~A} / \operatorname{sqrt}(\mathrm{Hz})$. 

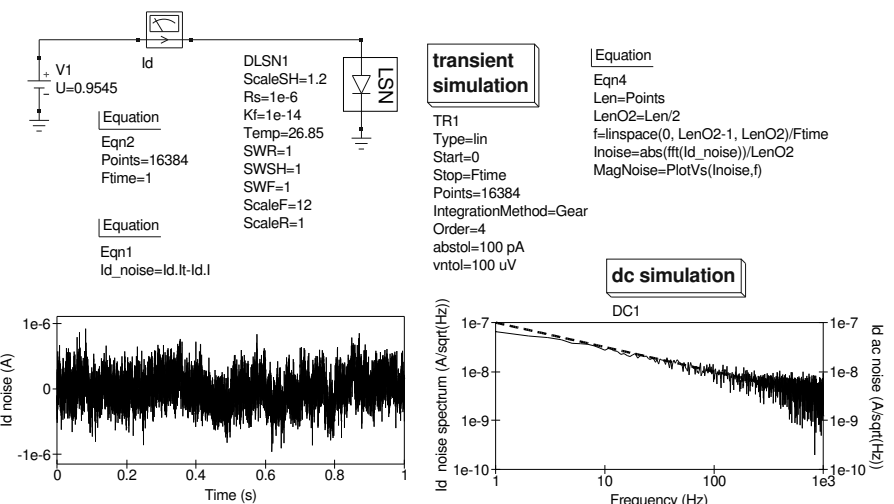

Figure 6. Time domain diode noise test circuit and simulation waveforms: frequency domain plot; solid line left hand scale, dashed line right hand scale.

\section{MODELLING BJT NOISE IN THE TIME DOMAIN}

A time domain noise model for an npn bipolar junction transistor (BJT) is illustrated in Figure 7. The noise contributions in this model are given by equations 4 and 5 respectively.

$$
\begin{gathered}
\bar{i}_{R B}^{2}(t)=\frac{4 \cdot k \cdot T}{R B} \cdot \triangle f \cdot \xi_{n}(t), \bar{i}_{R C}^{2}(t)=\frac{4 \cdot k \cdot T}{R C} \cdot \triangle f \cdot \xi_{n}(t) \bar{i}_{R E}^{2}(t)=\frac{4 \cdot k \cdot T}{R E} \cdot \triangle f \cdot \xi_{n}(t) \\
\bar{i}_{C}^{2}(t)=2 \cdot q \cdot I_{C} \cdot \triangle f \cdot \xi_{n}(t), \bar{i}_{B}^{2}(t)=2 \cdot q \cdot I_{B} \cdot \triangle f \cdot \xi_{n}(t)+K F \cdot \frac{I_{B}}{f^{A f}} \cdot \triangle f \cdot \xi_{f}(t)
\end{gathered}
$$

Where $I_{C}$ is the BJT dc collector current in $\mathrm{A}, I_{B}$ is the BJT dc base current in $\mathrm{A}, R c$ is the BJT series collector resistance in $\Omega, R B$ is the BJT series base resistance in $\Omega$, and $R E$ is the BJT series emitter resistance in $\Omega$. Unlike the diode time domain noise model shown in Figure 5 the npn BJT model has been optimized for run time speed by minimizing the number of circuit nodes, subcircuit blocks and standard components. The structure of the npn BJT model is arranged to allow easy conversion to Verilog-A code using the example shown in Table III as a template. Figure 8 gives a basic BJT noise test circuit and a set of time and frequency domain simulation waveforms. The latter being obtained using FFT techniques. To ensure that the noise current can flow in the BJT base lead a $50 \mathrm{k} \Omega$ resistor has been added to the test circuit, resulting in a base noise current that depends on the ratio of the $50 \mathrm{k} \Omega$ resistor and the value of $r_{\pi}$ in the BJT ac equivalent circuit at its dc operating point. The frequency domain plots show Qucs small signal ac noise analysis results superimposed on the noise data extracted from the npn BJT time domain noise model. The ac domain results were obtained using the same test circuit as the one shown in Figure 8. Comparison of the small signal ac and time domain collector current noise data indicates good agreement between both sets of noise data. This is very much expected as frequency dependent 1 /f noise dominates the collector current noise. A similar comparison of base current noise appear to suggest differences, at higher frequencies, between the small signal ac and time domain results. However, both sets of data plotted in Figure 8 are correct: at low frequencies 1/f noise again dominates yielding 


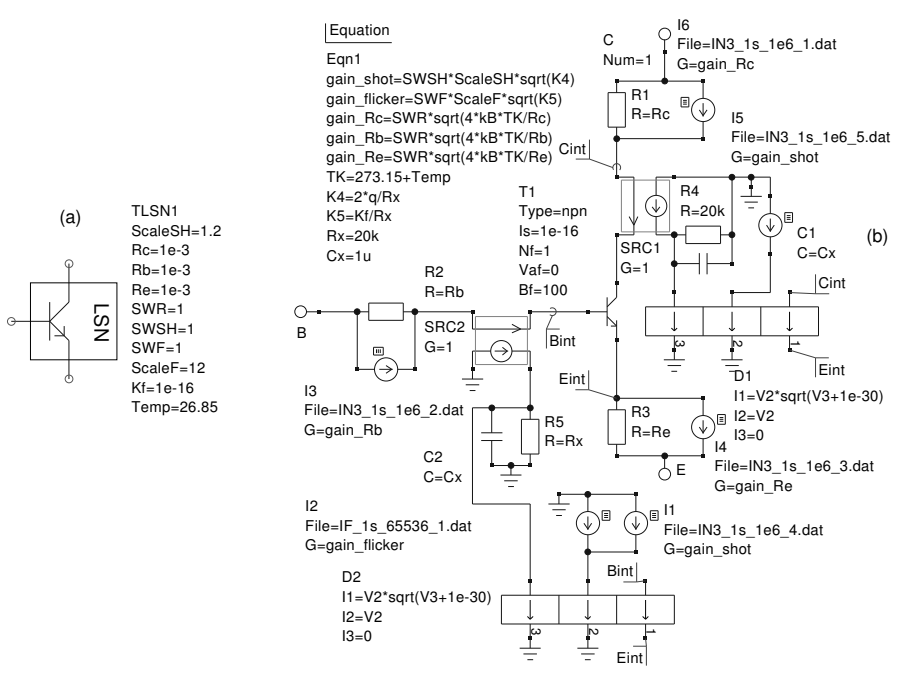

Figure 7. An npn BJT model with time domain noise additions: (a) Qucs schematic symbol; (b) model body.
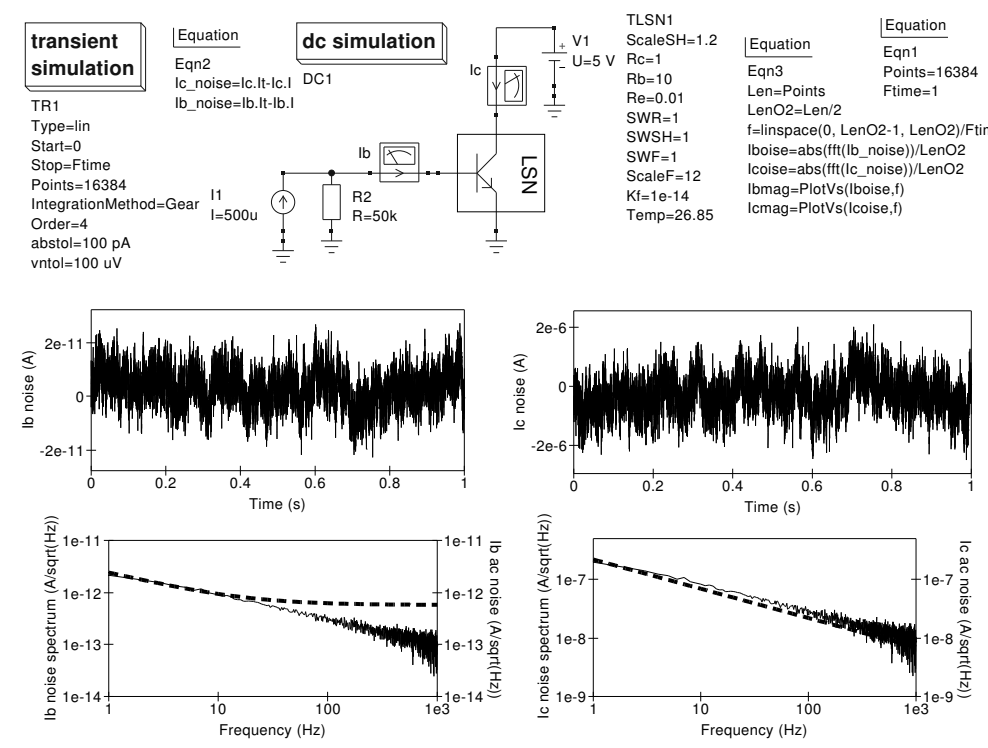

Figure 8. Time domain npn BJT noise test circuit and simulation waveforms: frequency domain plots; solid line left hand scales, dashed line right hand scales.

very similar noise values for both the ac and time domain data; at higher frequencies the $1 / \mathrm{f}$ noise declines until it eventually equals the base current shot noise in value. The small signal ac noise result show this occurs at around $50 \mathrm{~Hz}$. As shot noise is Gaussian distributed white noise its signal power is equally spread across all the FFT frequency bins (16384 in the test example) and hence it does not appear in the signal range plotted in Figure 8. 


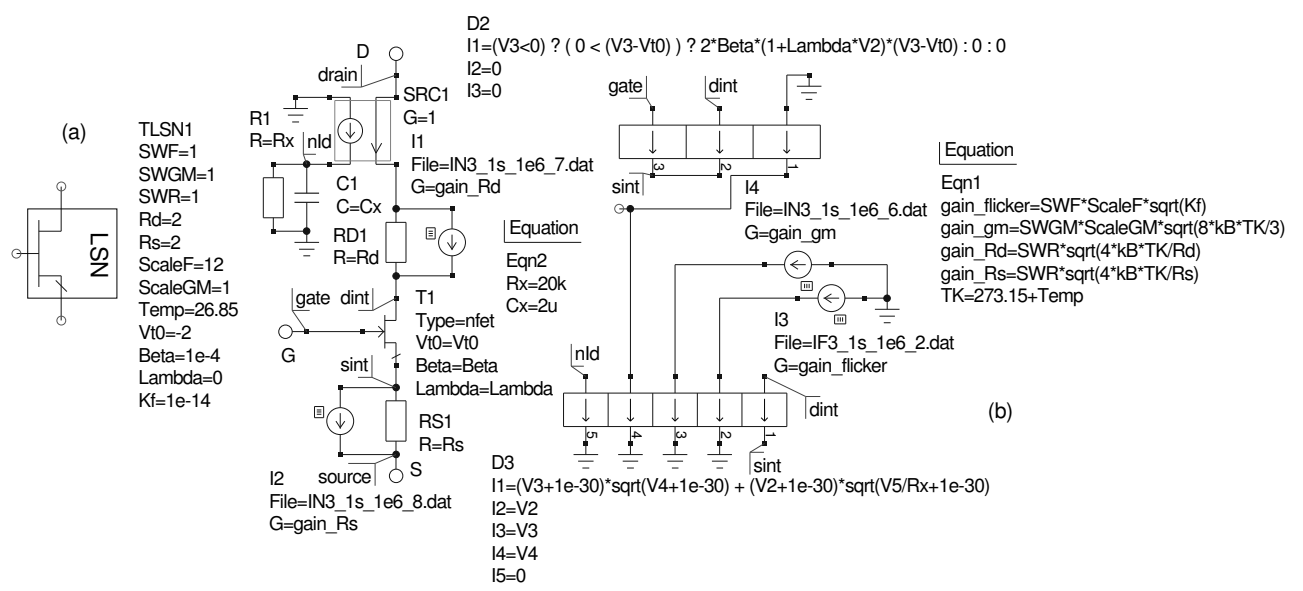

Figure 9. An $n$ type JFET model with time domain noise additions: (a) Qucs schematic symbol; (b) model body.

\section{MODELLING FET, MOSFET AND MESFET NOISE IN THE TIME DOMAIN}

FET, MOSFET and MESFET noise contributions are specified by equations 6 and 7 .

$$
\begin{aligned}
\bar{i}_{R D}^{2}(t) & =\frac{4 \cdot k \cdot T}{R D} \cdot \Delta f \cdot \xi_{n}(t), \bar{i}_{R S}^{2}(t)=\frac{4 \cdot k \cdot T}{R S} \cdot \Delta f \cdot \xi_{n}(t) \\
\bar{i}_{D}^{2}(t) & =\frac{8 \cdot k \cdot T \cdot g_{m}}{3} \cdot \Delta f \cdot \xi_{n}(t)+K f \cdot \frac{I_{D}}{f^{A f}} \cdot \Delta f \cdot \xi_{f}(t)
\end{aligned}
$$

Where $I_{D}$ is the drain to source dc current in A, $g_{m}$ is the transconductance of the device at the dc operating point, $R D$ is the series drain resistance in $\Omega$, and $R S$ is the series source resistance in $\Omega$. Figure 9 presents a time domain noise model for $n$ type FET devices. Qucs equation-defined device D2 models $g_{m}$, making the model slightly more complex than the diode and BJT time domain models. In Figure 9 the dc gate current is assumed to be zero or so small that it does not generate any significant noise components. Similarly, gate series resistance is not included in the model as it has negligible effect on the time domain noise properties of the device. A similar strategy is adopted with the standard SPICE FET model. A test circuit for determining FET noise is shown in Figure 10. In this example circuit a low pass RC filter is used to apply dc voltage to the gate of the FET under test. Without this filter the gate potential would be clamped at a constant dc voltage, causing malfunction of the test circuit. The waveforms illustrated in Figure 10 confirm that at low frequencies $1 / \mathrm{f}$ noise dominates the FET noise performance. Level one MOSFET and MESFET [7] time domain extended noise models are very similar to the FET model given in Figure 9. For completeness the body of a MOSFET and a MESFET time domain noise model are shown in Figure 11. 

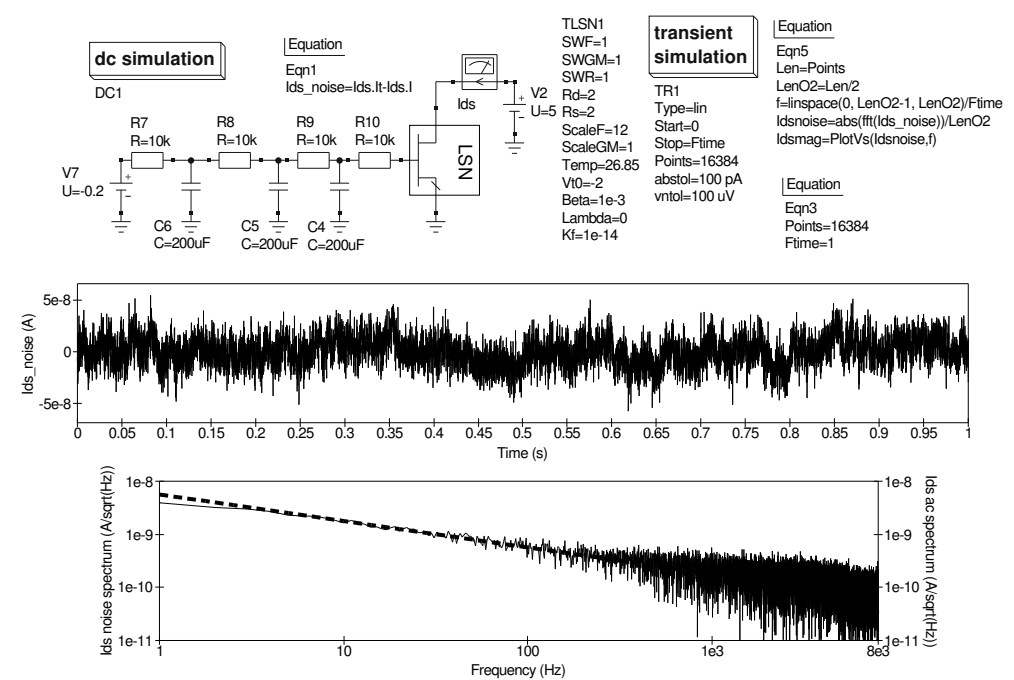

Figure 10. Time domain $\mathrm{n}$ type FET noise test circuit and simulation waveforms: frequency domain plots; solid line left hand scales, dashed line right hand scales.

\section{CONCLUSIONS}

Transient simulation of time domain noise is rarely undertaken by the current generation of circuit simulators. This paper introduces a tabular noise source approach to time domain noise modelling and analysis which is suitable for simulating noise generated by resistive components, semiconductor devices and integrated circuits. In the text the proposed modelling technique is introduced by a series of example model designs which add time domain noise to the standard SPICE diode, BJT, JFET, MOSFET and MESFET models. Experience with the new noise modelling technique has shown that it is suitable for use with any general purpose circuit simulator provided it can handle subcircuits, equation-defined devices (or Verilog-A code models) and a text files, or a net-list statement, for listing tabulated voltage and current source noise data. By combining the Qucs GPL simulator with the Octave GPL numerical mathematics package it becomes possible to easily generate pseudo random time domain noise data, allowing very realistic noise current or voltage waveforms to be added to transient circuit simulation, Good agreement was found between the noise results obtained by small signal ac and transient noise simulation.

\section{REFERENCES}

1. Newton AR, Pederson DO, Sangiovanni-Vincentelli A. SPICE Version 2g User's Guide. Department of Electrical Engineering and Computer Sciences, University of California, Berkeley, CA, 1981.

2. Johnson B, Quarles T, Newton AR, Pederson DO, Sangiovanni-Vincentelli A. SPICE3 Version 3f User's Manual. Department of Electrical Engineering and Computer Sciences, University of California, Berkeley, CA, 1992.

3. Wedge SW, Rutledge DB. Wave Techniques for Noise Modeling and Measurement. IEEE Transactions on Microwave Theory and Techniques, 1992; 40(11):2004-2012. 


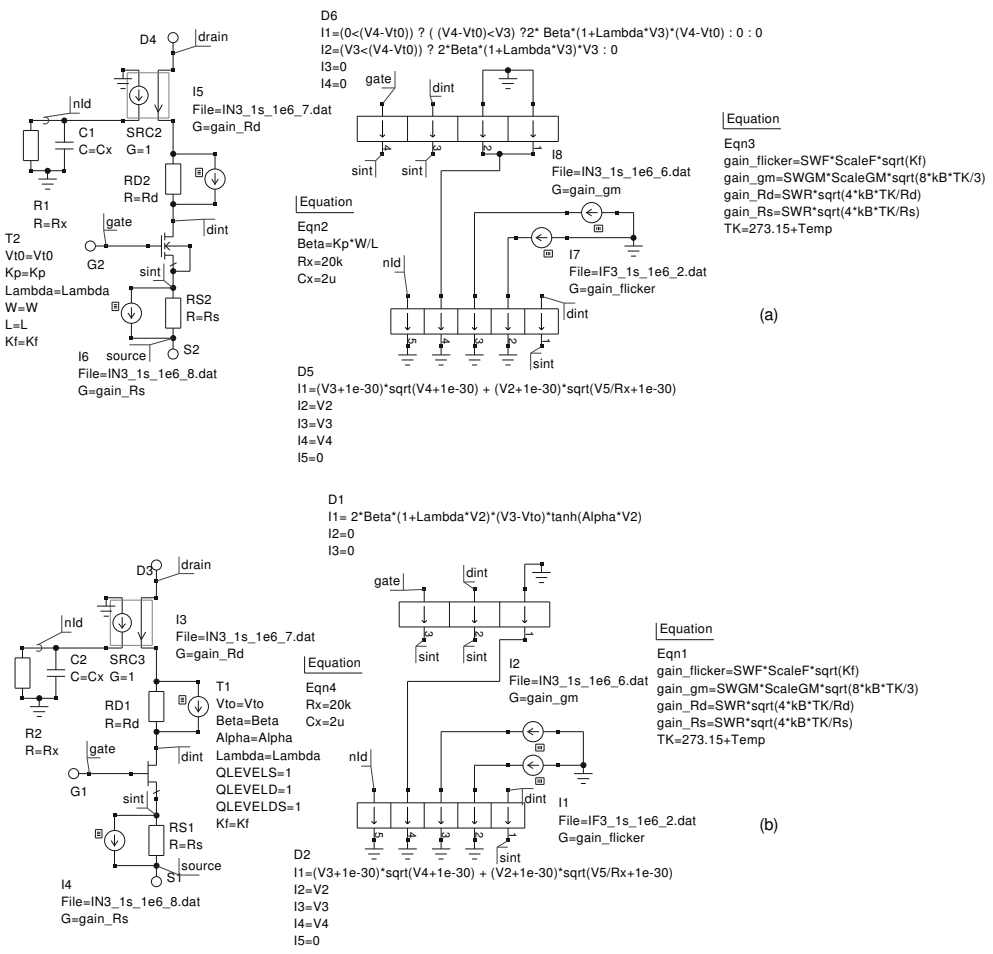

Figure 11. MOSFET and MESFET time domain noise models: (a) SPICE level 1 nMOSFET; (b) Curtice level 1 nMESFET.

4. Winkler R. Stochastic differential algebraic equations in transient noise analysis. Mathematics in Industry. (part 2), Springer, Berlin, 2008; 151-156.

5. Denk G, Winkler R. Modelling and simulation of transient noise in circuit simulation. Mathematical and computer modelling of dynamical systems, 2007; 13(4):383-394.

6. Fasce C, Massobrio G, Meda E, Profumo E. Semiconductor device modeling with SPICE. Antognetti P, Massobrio G.(eds). McGraw-Hill Book Company: New York, 1988; 289-309.

7. Brinson ME, Jahn S, Parruitte H. Verilog-A compact device models for GaAs MESFETs. Qucs: Report Book - Technical reports and descriptions, 2006. 1(5):88-127. Available from: http://sourceforge.net/docs/html [30 March 2010].

8. Margraf M, Jahn S, Flucke J, Jacob R, Habchi V, Ishikawa T, Gopala Krishna A, Brinson ME, Parruitte H, Roucaries B, Kraut G. Qucs (Quite universal circuit simulator). Version 0.0.15. 2010. Available from: http://sourceforge.net/index.html. [30 March 2010]

9. GNU Octave. 1992. Available from: http://www.gnu.orge/software/octave/ [30 March 2010]

10. Kraut G. Qucs File type voltage and current sources. 2007. Available from: http://sourceforge.net/docs/html [30 March 2010].

11. Jahn S, Margraf M, Habchi V, Jacob R. Appendix A: Qucs file formats. Qucs: Technical papers, 2003; 226-229. Available from: http://sourceforge.net/docs/html [30 March 2010].

12. Bell DA. Electrical Noise - Fundamentals and physical Mechanisms. D. Van Nostrand Company Ltd: London, 1960.

13. Blake K. OP AMP precision design: random noise. Microchip Technology Inc: Application note AN1228, 2008 Available from: http://ww1.microchip.com/downloads/en/AppNotes/01228a.pdf [30 March 2010].

14. Jahn S, Brinson ME. Interactive compact device modelling using Qucs equation-defined devices. International Journal of Numerical Modelling: Electronic Networks, Devices and Fields, 2008 21(3):335349

15. Accellera. Verilog-AMS Language Reference Manual, Version 2.2, 2004. Available from: 
http://www.accellera.org. [30 March 2010].

16. Little M, Powernoise.m MATLAB/Octave code for generating power law noise $1 / f^{\alpha}$. samp, University of Oxford, U.K. 20008. Available from http://www.eng.ox.ac.uk/samp/powernoise-soft.html. [31 March 2010]

17. Anderson B. Crest factor analysis for complex signal processing. RF integrated subsystems. www.rfdesign.com. Available from: http://www.mobiledevdesign.com/hardware/news/radio_crest_factor_analysis/. [11 April 2010]

\section{AUTHORS' BIOGRAPHIES}

M.E. Brinson received a first class honours BSc degree in the Physics and Technology of Electronics from the United Kingdom Council for Academic Awards in 1965, and a $\mathrm{PhD}$ in Solid State Physics from the University of London in 1968. Since 1968 Dr Brinson has held

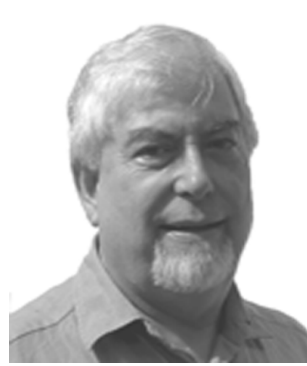
academic posts in Electronics and Computer Science. From 1997 to 2000 he was a visiting Professor of Analogue Microelectronics at Hochschule, Bremen, Germany. Currently, he is a visiting Professor at the Center for Communication Technology, London Metropolitan University, UK. $\mathrm{He}$ is a Chartered Engineer (CEng) and a Fellow of the Institution of Engineering and Technology (FIET), a Chartered Physicist (CPhys) and a Member of the Institute of Physics (MinstP). Dr Brinson joined the Qucs project development team in 2006, specializing in device and circuit modelling, testing and document preparation.

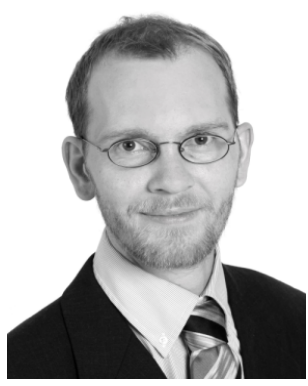

S. Jahn received a diploma in Electrical Engineering from the Technical University of Berlin in 2005. His specialist interests include highfrequency engineering and opto electronics. He completed his diploma thesis on EMC of integrated circuits while at Infineon Technologies AG. Stefan joined the Qucs project in 2003, specializing in circuit simulator development.

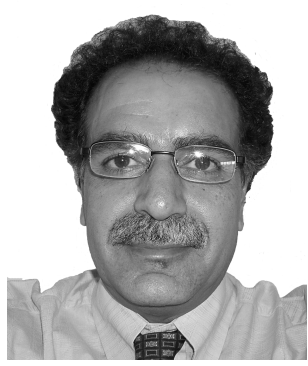

H. Nabijou received his BSc in Electronic Communications, his MSc in Digital Systems and Instrumentation and his $\mathrm{PhD}$ in Statistical Signal Processing from UK universities. His research interests include modelling of non-linear stochastic systems. He is currently an academic member of staff in the Faculty of Computing, and a member of the Center for Communication Technology, London Metropolitan University, UK. He is a member of the IEEE and a fellow of the Royal Statistical Society. Dr Nabijou has recently joined the Qucs Development Project, as an associate, working on the modelling of signal processing components and algorithms. 